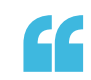

systemic injection of E. coli proteins ... decreased food intake and activated several anorexigenic pathways in the brains of rats

profile of the host; however, it is not

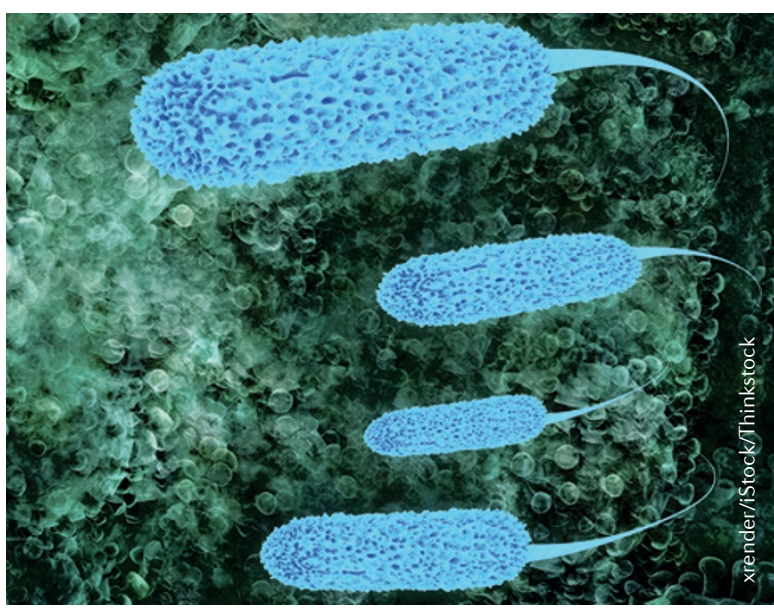

A new study has demonstrated that the appetites of rats are influenced by proteins released by Escherichia coli. The authors of the study suggest that these proteins act on the anorexigenic pathways in the brain.

The composition of the gut microbiota can affect the metabolic profile of the host; however, it is not known whether the gut microbiota

GUT MICROBIOTA

\title{
Proteins released by E. coli in the gut influence host appetite control
}

can influence appetite. To investigate this association, Sergueï Fetissov and co-workers focused on chaperone protein ClpB. This protein is a mimetic of $\alpha-\mathrm{MSH}$ (a peptide hormone known to induce satiety) that is released by E. coli.

A proteomics analysis revealed that E. coli express different proteins in the different growth phases. In particular, expression of $\mathrm{ClpB}$ was increased during the stationary growth phase, compared with the exponential growth phase.

Infusion of a nutritional culture medium into the colons of rats induced bacterial proliferation in the gut, an effect that was not seen when water was infused. The researchers were also able to demonstrate that $\mathrm{ClpB}$ was present in the plasma of rats.

Importantly, systemic injection of $E$. coli proteins from bacteria in the stationary phase decreased food intake and activated several anorexigenic pathways in the brains of rats. In addition, direct application of $\mathrm{ClpB}$ to the brain activated $\alpha-\mathrm{MSH}$ neurons. Thus, "plasmatic bacterial proteins, including $\mathrm{ClpB}$, might represent a molecular link between gut bacteria and the host that has a long-term modulatory role in appetite control," explains Fetissov.

The researchers are now planning to study whether the stimulation of satiety pathways by the gut microbiota is insufficient in obesity and, if so, whether this deficiency can be corrected. "Another implication of this study is a possible relationship between bacterial growth phases and binge eating in the host; according to our results, continuous nutrient-induced bacterial growth might delay bacterially induced host satiety," concludes Fetissov.

Claire Greenhill

ORIGINAL ARTICLE Breton, J. et al. Gut commensal E. coli proteins activate host satiety pathways following nutrient-induced bacterial growth.Cell Metab.http://dx.doi.org/10.1016/j cmet.2015.10.017 